\title{
Synchronization of Hybrid Microgrids with Communication Latency
}

\author{
Jingang Lai, ${ }^{1}$ Hong Zhou, ${ }^{1}$ Wenshan Hu, ${ }^{1}$ Xiaoqing Lu, ${ }^{2}$ and Liang Zhong ${ }^{1}$ \\ ${ }^{1}$ Department of Automation, Wuhan University, Wuhan 430072, China \\ ${ }^{2}$ College of Electrical and Information Engineering, Hunan University, Changsha 410082, China \\ Correspondence should be addressed to Wenshan Hu; wenshan.hu@whu.edu.cn
}

Received 11 November 2014; Revised 23 January 2015; Accepted 23 January 2015

Academic Editor: Hui Zhang

Copyright (c) 2015 Jingang Lai et al. This is an open access article distributed under the Creative Commons Attribution License, which permits unrestricted use, distribution, and reproduction in any medium, provided the original work is properly cited.

\begin{abstract}
A distributed cooperative control scheme is proposed in order to implement a distributed secondary control for hybrid lossy microgrids. The designed distributed control is able to synchronize the frequency of inverse-based distributed generators (DGs) and minisynchronous generators (MSGs/SGs) to the desired state with a virtual leader DG/SG (reference value) in a distribution switching network under the existence of time-varying communication delays. The secondary control stage selects suitable frequencies of each DG/SG such that they can be synchronized at the desired set point. Using the proposed algorithm, each DG/SG only needs to communicate with its neighboring DGs/SGs intermittently even if the communication networks are local, the topology is time-varying, and the communication delays may exist. Therefore, the failure of a single DG/SG will not produce the failing down of the whole system. Sufficient conditions on the requirements for the network connectivity and the delays boundedness which guarantees the stability and synchronization of the controlled hybrid lossy microgrid power systems are presented. The feasibility of the proposed control methodology is verified by the simulation of a given lossy microgrid test system.
\end{abstract}

\section{Introduction}

The recent changes in the structure of power generation towards a distributed generation have motivated the increasing interest in the control of so-called microgrids. As the main building blocks of smart grids, microgrids are small-scale power systems that facilitate the effective integration of highly hybrid and heterogeneous DGs and storage devices including solar (photovoltaic array), wind, microturbines, supercapacitor, and batteries $[1,2]$. The structure of hybrid microgrid is as shown in Figure 1. Many of these energy sources and storage devices generate or reserve variable frequency $\mathrm{AC} / \mathrm{DC}$ power and are interfaced with a synchronous $\mathrm{AC}$ grid via power electronic DC/AC inverters [3]. Meanwhile, with the rapid growth of power electronics techniques on MSGs or virtual SGs, the increasing application of clear energy sources, including diesel plants, wind plants, and geothermal plants, also makes them possible to be integrated into microgrids. Therefore, new control strategies on how to preserve the synchronization and proportional power sharing in the connected or isolated manner are demanded for the network [4].

Recently, hierarchical control for microgrids has been proposed in order to standardize their operation and functionalities, that is, primary control [5] (droop control maintaining voltage and frequency stability of the microgrid subsequent to the islanding process), secondary control [6] (compensating the voltage and frequency derivations caused by primary control), and tertiary control [7] (optimal operation in both operating modes and power flow control in grid-connected mode).

However, the conventional secondary control of microgrids assumes a centralized control structure which requires a complex communication network and a central controller. A central controller reduces the system reliability [8]. Additionally, some literatures point that physical and communication structures of microgrid can be time-varying because of the desired plug-and-play capability of microgrids [9]. From 


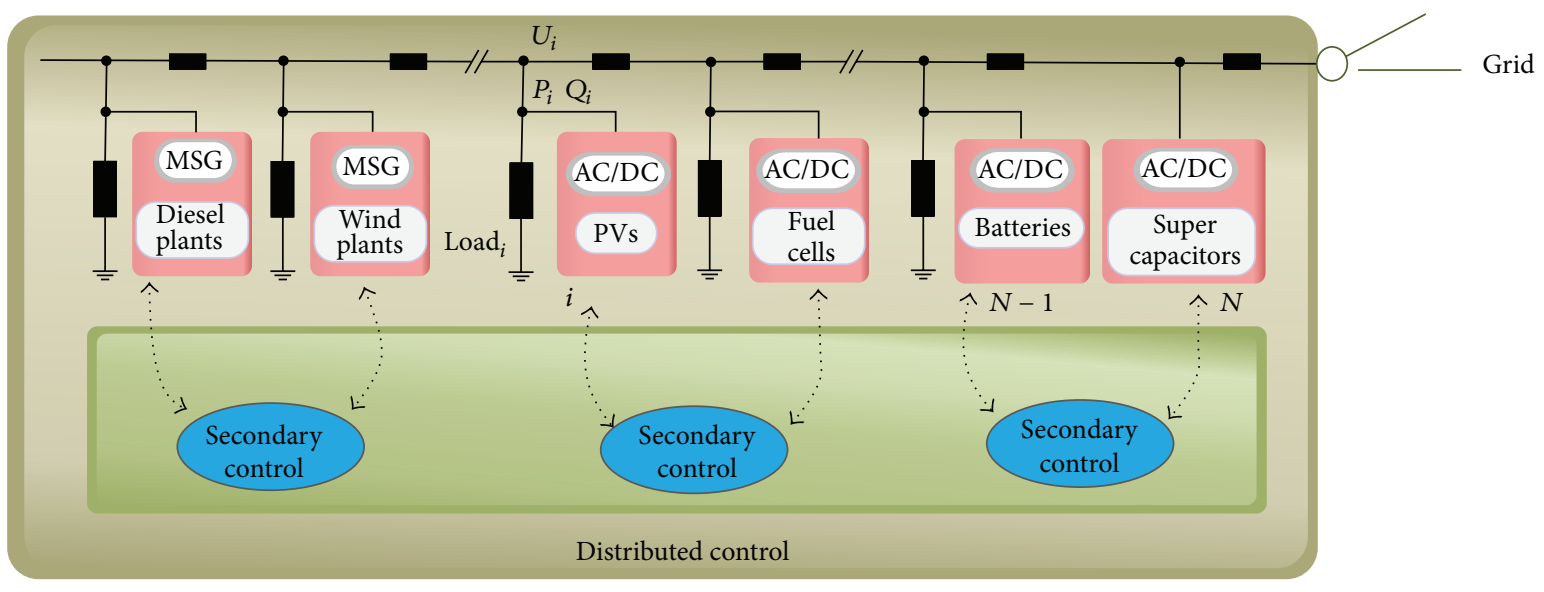

Large-scale heterogeneous microgrids

FIGURE 1: The structure of hybrid microgrid.

this perspective, the distributed control structure provides a robust secondary control framework $[10,11]$. It could largely reduce the system reliability $[12,13]$. In contrast, decentralised control structures are adopted in $[14,15]$ to avoid the above drawbacks. Nevertheless, for the large-scale microgrids, it is impossible to determine and maintain a feasible operating condition if all of the DGs are running independently. Alternatively, affected by the idea of cooperation control for multiagent systems [16-21], there are increasing researchers who begin to focus on the distributed control structures which allow all the DGs to communicate with their neighbors and share information among neighboring units via local sparse communication networks [22-26].

Particularly, in paper [27], the authors proposed a secondary voltage and frequency control scheme based on the distributed cooperative control of multiagent systems and thus improved the system reliability. In order to regulate the power output of a microgrid dynamics consisting of a large amount of photovoltaic generators, [28] presented a selforganizing strategy in a distributed time-varying network.

The main common features of the above literatures lie in that a distributed communication network has been taken as a basis for the control strategies designed. In this case, the network variability and communication delays are usually unavoidable. They cannot be neglected for the system stability analysis [29, 30]. In fact, in normal circumstances, the microgrids frequency fluctuation and the amplitudes of each DG unit response to primary frequency control are very small. For the purpose of efficient signal transmission, it is inevitable to induce some disturbance and time-delays during the signal processing. Hence, in [29], decentralized inverter control algorithms for power sharing with nonnegligible constant communication delays are investigated. Also, by using the small-signal analysis method, a distributed secondary voltage and frequency control for reactive power sharing are discussed in [30], in which the constant communication delays are analyzed only in an experimental simulation way.
In view of the above analysis, most research on synchronization and power sharing of microgrids has focused on purely inverter-based systems. However, from a practical consideration, most present and near-future applications involve networks of mixed generation mode including SGs and inverter-based DGs and/or storage devices [31]. This paper aims to develop a distributed cooperative control scheme for hybrid microgrids that is able to synchronize the frequency of DGs/SGs to the desired state with a virtual leader DG/SG (reference value) in a distribution switching network under the existence of time-varying communication delays. Combining with the conventional droop control technique, the distributed cooperative control algorithm is designed and deployed into the secondary control stage, in which manner the control derivation produced during the primary control stage can be well restored. And the secondary control stage selects suitable frequencies of each DG/SG such that they can synchronize to the desired state. With the proposed algorithms, each DG/SG only needs to communicate with its neighboring DGs/SGs intermittently even if the communication networks are local, the topology is time-varying, and the communication delays may exist. Sufficient conditions on the requirements for the network connectivity and the delays boundedness that guarantees the stability and reliability of the controlled microgrid power systems are presented. The effectiveness of the proposed control methodology is verified by the simulation of a lossy microgrid test system.

The main contributions of this paper lie in the following aspects. Firstly, in order to restore the frequency in hybrid lossy microgrids, one first designs a distributed secondary control algorithm to synchronize the frequency of each DG/SG to the desired state (reference value $50 \mathrm{~Hz}$ ). Secondly, the considered distributed communication network is local and time-varying and with time-varying communication delays. By Lyapunov-Krasovskii stable analysis technique, sufficient conditions on the requirements for the network connectivity and the delays boundedness that guarantees 
the stability and synchronization of the controlled microgrid power systems are presented.

The rest of this paper is organized as follows. In Section 2, the droop-based distributed cooperative control for microgrids problem is formulated and several necessary lemmas are then given. The distributed cooperative algorithm is designed for realizing the frequency restoration in Section 3. Section 4 gives some typical examples to verify the effectiveness of the proposed algorithm. Finally, some concluding remarks are drawn in Section 5.

\section{Problem Formulation}

In normal situation, the microgrid is operating with the nominal frequency and voltage magnitudes. The objective of primary control is to maintain the frequency and/or voltage magnitudes near around its nominal value once user demands and/or power supply changes. Herein our attentions are restricted to the frequency regulation, so the inverters are considered as voltage source with constant voltage amplitude. And we adopt the physically reasonable and widely adopted assumption of an inductive microgrid [26].

The procedure of primary and secondary control is as shown in Figure 2. In the steady state, the load power curve $P_{L}$ (after linearization processing) and the generator power curve $P_{G}$ (after linearization processing) have a balance point at $a$, whose coordinate is $\left(P^{\text {nom }}, \omega^{\text {nom }}\right)$. The frequency $\omega$ will decrease by $\Delta \omega$ in order to make the output power of the DG/SG increase to the point $c$. Due to the inherent defects of primary control, the increase of the generator power $\Delta P^{\prime}$ in the above case is smaller than the actual increase in the load power $\Delta P$. In order to compensate the frequency deviation produced by the primary control, secondary frequency control (the green lines in Figure 2) is deployed to regulate the frequency $\Delta \omega$ such that the intersection point $c$ moves to $d$.

2.1. Droop Characters of SGs in Electrical Networks. For the dynamics of SGs, we consider the conventional expression of a regulated SG by a constant voltage behind transient reactance [24]. The so-called regulated machine is always equipped with a speed governor connected to a governing system. The speed governor is controlled to adjust the output of turbine mechanical power $P_{i}^{M}$ and its speed. Droop control is the intrinsic characteristic to maintain system stability. Here, we assume that there is an approximate linear relationship between the valve position and the mechanical power. It is worth noting that the mechanical speed $\omega^{M}$ is different from the electrical speed $\omega$, but their relationship is $\omega=(n / 2) \omega^{M}$, where $n$ is the number of machine poles [31]. Droop control eventually can be expressed as follows:

$$
P^{M}(t)=P^{M, \text { nom }}-\frac{1}{\widehat{k}_{P}} \omega_{i}(t)
$$

where $\omega$ is an input signal, $P^{M}$ is an output signal, and $1 / \widehat{k}_{P}$ denotes the droop coefficient. The $P^{M, n o m}$ is a constant and the nominal set point for the mechanical power.

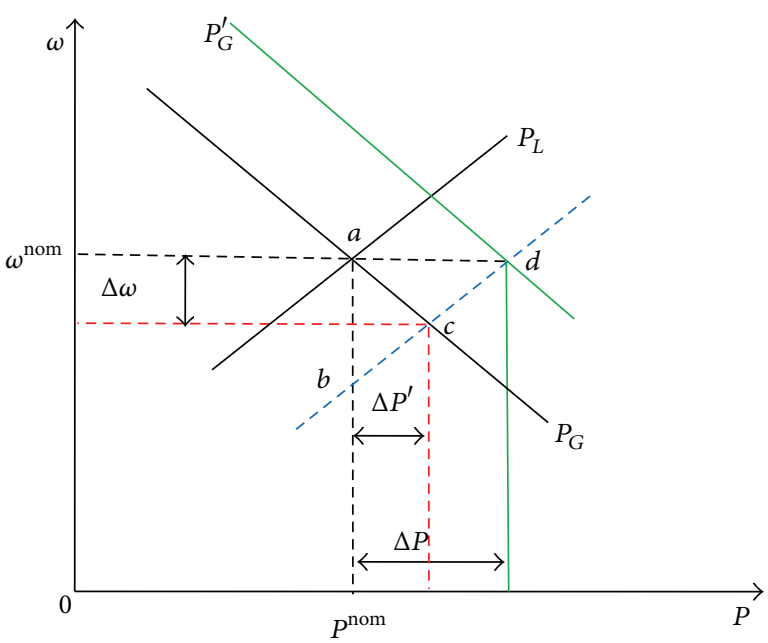

FIGURE 2: Primary and secondary frequency regulation.

2.2. Droop Control for Inverter-Based DGs Networks. From the above theoretical analysis, the droop technique deployed in primary controller for DGs during the multiple loop control process can be given by

$$
\omega=\omega^{\text {nom }}-K^{P}\left(P-P^{\text {nom }}\right)
$$

where $\omega^{\text {nom }}$ is chosen from the nominal set point of frequency of the DG unit, $P$ is the measured active powers at the DG terminal, $K^{P}$ is the associated droop coefficient that is usually selected based on the active power rating, and $P^{\text {nom }}$ is chosen from the nominal set point of active power of the DG unit.

The control process of each DG generally consists of three control loops (as shown in Figure 3), that is, the power controller, voltage magnitudes controller, and current controller.

The active power $P_{i}$ and reactive power $Q_{i}$ of the $i$ th DG are calculated based on the low-pass filter (with cutoff frequency $\omega_{i}^{c}$ ), measured output voltage magnitude, and output current. They can be described by the following equations, respectively:

$$
\begin{aligned}
P_{i} & =\frac{\omega_{i}^{c}}{\omega_{i}^{c}+s}\left(V_{i}^{\mathrm{od}} I_{i}^{\mathrm{od}}+V_{i}^{\mathrm{oq}} I_{i}^{\mathrm{oq}}\right), \\
Q_{i} & =\frac{\omega_{i}^{c}}{\omega_{i}^{c}+s}\left(V_{i}^{\mathrm{od}} I_{i}^{\mathrm{oq}}-V_{i}^{\mathrm{oq}} I_{i}^{\mathrm{od}}\right) .
\end{aligned}
$$

The nominal value of output frequency $\omega_{i}^{\text {nom }}$ of the power controller is used by the sinusoidal pulse width modulation (SPWM) inverter as frequency reference, while the voltage reference of the SPWM inverter needs to be regulated by the following voltage and current controllers. Based on the references provided by the power controller, $V_{i}^{\text {od,nom }}$ and 


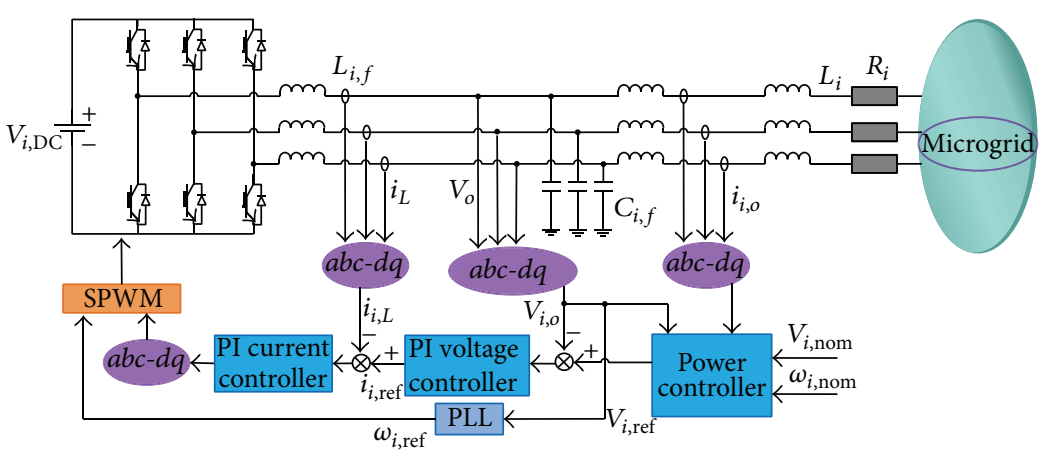

FIgURE 3: The multiple loop control schematic of the inverter in microgrids.

$V_{i}^{\mathrm{oq}, \text { nom }}$, the output of the voltage magnitude controller is given by

$$
\begin{aligned}
I_{i}^{\mathrm{od}, \mathrm{nom}}= & k_{i}^{P V}\left[V_{i}^{\mathrm{od}, \mathrm{nom}}-V_{i}^{\mathrm{od}}\right] \\
& +k_{i}^{I V} \int\left[V_{i}^{\mathrm{od}, \mathrm{nom}}-V_{i}^{\mathrm{od}}\right] \mathrm{d} t-\omega_{i}^{\mathrm{nom}} C_{f} V_{i}^{\mathrm{oq}} \\
& +F I_{i}^{\mathrm{od}}, \\
I_{i}^{\mathrm{oq}, \mathrm{nom}}= & k_{i}^{P V}\left[V_{i}^{\mathrm{oq}, \mathrm{nom}}-V_{i}^{\mathrm{oq}}\right] \\
& +k_{i}^{I V} \int\left[V_{i}^{\mathrm{oq}, \mathrm{nom}}-V_{i}^{\mathrm{oq}}\right] \mathrm{d} t-\omega_{i}^{\mathrm{nom}} C_{f} V_{i}^{\mathrm{od}} \\
& +F I_{i}^{\mathrm{oq}},
\end{aligned}
$$

where $k_{i}^{P V}$ and $k_{i}^{I V}$ are the proportional and integral gains of the voltage magnitudes controller of $i$ th DG, respectively, while $C_{f}$ and $F$ are the capacitance of the LC filter and feedforward gain, respectively. On the other hand, based on the references provided by the voltage magnitudes controller, $I_{i}^{\text {od,nom }}$ and $I_{i}^{\text {oq, nom }}$, the output of the current controller is given by

$$
\begin{aligned}
V_{i}^{\mathrm{od}, \mathrm{nom}}= & k_{i}^{P I}\left[I_{i}^{\mathrm{od}, \mathrm{nom}}-I_{i}^{\mathrm{od}}\right]+k_{i}^{\mathrm{II}} \int\left[I_{i}^{\mathrm{od}, \mathrm{nom}}-I_{i}^{\mathrm{od}}\right] \mathrm{d} t \\
& -\omega_{i}^{\mathrm{nom}} L_{f} I_{i, L}^{\mathrm{oq}}, \\
V_{i}^{\mathrm{oq}, \mathrm{nom}}= & k_{i}^{P I}\left[I_{i}^{\mathrm{oq}, \mathrm{nom}}-I_{i}^{\mathrm{oq}}\right]+k_{i}^{I I} \int\left[I_{i}^{\mathrm{oq}, \mathrm{nom}}-I_{i}^{\mathrm{oq}}\right] \mathrm{d} t \\
& +\omega_{i}^{\mathrm{nom}} L_{f} I_{i, L}^{\mathrm{od}}
\end{aligned}
$$

The dynamics of a frequency droop-controlled inverter (2) and a regulated SG (1) are equivalent (see [9] for details on relevant proof). Therefore, next on the analysis of the droop control, DGs and SGs will be considered to be of the same features and deploy the same dynamic model.

Since inverters may connect pure storage devices, for example, batteries, to the network, $P_{i}^{\text {nom,stor }}$ can also take negative values. In that case, the storage device is charged in dependency of the excess power available in the network and thus functions as a frequency dependent load.
2.3. Secondary Control for Hybrid Microgrid. The conventional secondary control is a kind of centralized control methods, the nominal value $\omega^{\text {nom }}$ is used in the primary control stage, and it will be regulated gradually in each cycle of secondary control process. Note that the final desired frequency value can be measured from the main grid in the connected mode or obtained by command generator or many forms of DG/SG such as fuel-cells and microturbines in the islanded mode.

Different from the conventional secondary control with centralized mode, this paper in the secondary frequency control stage proposes one distributed cooperative control algorithm for each DG/SG. The basic idea of the proposed distributed control is by incorporating local communication networks that share information among neighboring units.

From this perspective, a microgrid can be considered as a multiagent system, where each DG/SG is an agent. The secondary control design for frequency resembles a tracking synchronization problem while that design for proportional power sharing is carried out. For this purpose, the important network digraph theory is introduced in the following.

2.4. Graph Theory. In this paper, the required communication network for microgrids can be modeled by a digraph $\mathscr{G}(\mathscr{V}, \mathcal{E}, \mathscr{A})$, where the node set $\mathscr{V}=\left\{v_{1}, v_{2}, \ldots, v_{N}\right\}$ denotes the set of DGs/SGs, the set of edges $\varepsilon \subseteq \mathscr{V} \times \mathscr{V}$ denotes the communication links among DGs/SGs, and $\mathscr{A}=\left(a_{i j}\right)_{N \times N}$ is a weighted adjacency matrix defined as $a_{i i}=0$ and $a_{i j} \geq 0$. $a_{i j}>0$ if and only if the edge $\left(v_{i}, v_{j}\right) \in \varepsilon$. The set of neighbors of the $i$ th DG/SG $v_{i}$ is given by $N_{i}=\left\{v_{j} \in \mathscr{V}:\left(v_{i}, v_{j}\right) \in \varepsilon\right\}$. The degree matrix is defined as $D=\operatorname{diag}\left\{d_{1}, \ldots, d_{N}\right\}$ with $d_{i}=\sum_{j \in N_{i}} a_{i j}$, and then $L=D-A$ is the Laplacian matrix, which has all row sums equal to zero; that is, $L 1_{N}=0$, with $1_{N}=(1, \ldots, 1)^{T} \in R^{N}$.

In the following, the digraph $\overline{\mathscr{G}}$ is used to describe the interconnection topology of a microgrid consisting of one virtual leader DG/SG, denoted by 0 , and followers DGs/SGs, denoted by $1, \ldots, N$. Diagonal matrix $B=\operatorname{diag}\left\{a_{10}, \ldots, a_{N 0}\right\}$ is called the leader adjacency matrix, where $a_{i 0}>0$ if follower DG/SG $v_{i}$ is connected to the leader across the communication link $\left(v_{0}, v_{i}\right)$; otherwise $a_{i 0}=0$. The following lemma plays an important role in the proof of our main results. 
Lemma 1 (see [32]). If the digraph $\mathscr{G}$ corresponding to the Laplacian matrix $L$ is strongly connected, then (i) if $q=$ $\left(q_{1}, \ldots, q_{N}\right)^{T}$ is a left eigenvector of $L$ corresponding to eigenvalue 0 , then $q_{i}>0$ holds for all $i=1, \ldots, N$, and (ii) all the eigenvalues of $L+B$ have positive real parts, where $B=$ $\operatorname{diag}\left\{b_{1}, \ldots, b_{N}\right\}, \sum_{i=1}^{N} b_{i}>0$, and $b_{i} \geq 0$ for all $i=1, \ldots, N$.

\section{Main Results}

In this section, one will discuss the distributed cooperative control schemes for frequency and power sharing in Sections 3.1 and 3.2 , respectively.

It should be noted that the dynamics of the voltage and current control loops are much faster than the dynamics of the power control loop [30]. Therefore, it is necessary and intentional to design a typical inverter system by applying the time separation operation [6]. In order to facilitate our analysis, this paper will focus on the stability of power control loop, while the investigation of current controllers follows traditional proportional-integral (PI) controllers.

3.1. Distributed Cooperative Control for Frequency. In this paper, the $d-q$ reference frame transformation is considered, where the $d$-axis and $q$-axis of the reference frame of each inverter are rotating at the common reference frequency. Based on the traditional droop control strategy, the reference of frequency of the $i$ th inverter-based DG or SG is determined by the power control loop and can be abstracted as

$$
\omega_{i}=\omega_{i}^{\mathrm{nom}}-K_{i}^{P}\left(P_{i}-P_{i}^{\mathrm{nom}}\right),
$$

where $\omega_{i}^{\text {nom }}$ is the nominal set point of output frequency and $P_{i}^{\text {nom }}$ is the nominal set point of output active, respectively, while $K_{i}^{P}$ is the frequency droop coefficient.

In the following, a distributed pinning control algorithm is designed to pin the frequency $\omega_{i}$ of DGs/SGs to synchronize the reference frequency $w^{\text {ref }}$, respectively.

According to the thought of so-called input-output feedback linearisation [27], differentiating the equations in (6) yields

$$
\dot{\omega}_{i}=\dot{\omega}_{i}^{\text {nom }}-K_{i}^{P} \dot{P}_{i}=u_{i}^{\omega},
$$

where $u_{i}^{\omega}$ is the pinning controllers which will be designed later such that $\lim _{t \rightarrow+\infty}\left|\omega_{i}(t)-\omega^{\mathrm{ref}}(t)\right|=0$ for $i=1, \ldots, N$.

Then the nominal control input $\omega_{i}^{\text {nom }}$ used in the droop control procedure can be computed by the following integrator:

$$
\omega_{i}^{\text {nom }}=\int\left(u_{i}^{\omega}+K_{i}^{P} \dot{P}_{i}\right) \mathrm{d} t .
$$

Since it is assumed that each DG/SG only needs to communicate with its neighboring DGs/SGs through a communication network, the pinning controllers $u_{i}^{\omega}$ are designed based on the own information of each DG/SG and the information of its neighbors:

$$
u_{i}^{\omega}=\sum_{j \in N_{i}(t)} a_{i j}(t)\left[\omega_{j}(t-\tau(t))-\omega_{i}(t-\tau(t))\right]
$$

for $i=1, \ldots, N$, where the time-varying delay $\tau(t) \geq 0$ is a continuously differentiable function and the factors of adjacency matrix $a_{i j}(t)$ are also allowed to be dynamically changing.

In order to facilitate analysis, some denotations are given. Let $B=\operatorname{diag}\left\{a_{10}, \ldots, a_{N 0}\right\}, e_{\xi}(t)=\xi(t)-\bar{\xi}(t)$ with $\xi=$ $\left(\omega_{1}, \ldots, \omega_{N}\right)^{T} \in R^{N}$ and $\bar{\xi}=\left(\omega^{\mathrm{ref}} 1_{N}\right)^{T} \in R^{N}$; then combining (7) and (9) and the fact that $\dot{\omega}^{\text {ref }}=0$, one can deduce the following error system:

$$
\begin{aligned}
\dot{e}_{\xi}(t) & =-\left(H_{s} \otimes I_{2}\right) e_{\xi}(t-\tau(t)), \\
s & =\sigma(t),
\end{aligned}
$$

where $H_{s}=L_{s}+B_{s}$ with the Laplacian matrix $L_{s}$ of digraph $\mathscr{G}_{s}, \otimes$ denotes the Kronect product, $I_{2}$ denotes the twodimensional identity matrix, and $\sigma(t):[0,+\infty) \rightarrow \rho_{\Gamma}=$ $\{1, \ldots, m\}\left(m \in Z^{+}\right.$denotes the total number of all possible directed graphs) is a switching signal that determines the communication topology $\mathscr{G}$. If $\sigma(t)$ is a constant function, then the corresponding topology is fixed.

Theorem 2. Suppose that the digraph $\mathscr{G}_{s}$ is always strongly connected and there exists at least one $i \in\{1, \ldots, N\}$ such that $a_{i 0}>0$ for any switching signal $s=\sigma(t)$. If the time-varying delays $\tau(t)$ satisfy $\tau(t)<d$ and $\dot{\tau}(t)<d_{1}<1$, then the distributed controllers (9) can pin the DG/SG output frequency $\omega_{i}$ to the reference states $\omega^{\text {ref }}$ asymptotically.

Proof. Define a common Lyapunov-Krasovkii function for system (10) as follows:

$$
\begin{aligned}
V(t)= & e_{\xi}^{T}(t)\left(\Xi \otimes I_{2}\right) e_{\xi}(t) \\
& +\int_{-d}^{0} \int_{t+\theta}^{t} \dot{e}_{\xi}^{T}(r) \dot{e}_{\xi}(r) \mathrm{d} r \mathrm{~d} \theta \\
& +\gamma \int_{t-\tau(t)}^{t} e_{\xi}^{T}(r)\left[\left(H_{s}^{T} H_{s}\right) \otimes I_{2}\right] e_{\xi}(r) \mathrm{d} r
\end{aligned}
$$

where $\Xi=\operatorname{diag}\left\{q_{1}, \ldots, q_{N}\right\}$ with the positive left eigenvector $q=\left(q_{1}, \ldots, q_{N}\right)^{T}$ corresponding to the zero eigenvalue of the Laplacian matrix $L_{s}$. Note that since $\mathscr{G}_{s}$ is strongly connected, one can always choose $q$ such that $\sum_{i=1}^{N} q_{i}=1$ and $q_{i}>0$ for all $i=1, \ldots, N$ (by Lemma 1 ).

Along the trajectory of system (10), we have

$$
\begin{aligned}
\dot{V}(t)= & -2 e_{\xi}^{T}(t)\left[\left(\Xi H_{s}\right) \otimes I_{2}\right] e_{\xi}(t-\tau(t))+\gamma e_{\xi}^{T}(t) \\
& \cdot\left[\left(H_{s}^{T} H_{s}\right) \otimes I_{2}\right] e_{\xi}(t)-\int_{t-d}^{t} \dot{e}_{\xi}^{T}(r) \dot{e}_{\xi}(r) \mathrm{d} r \\
& -\gamma(1-\dot{\tau}(t)) e_{\xi}^{T}(t-\tau(t))\left[\left(H_{s}^{T} H_{s}\right) \otimes I_{2}\right] \\
& \cdot e_{\xi}(t-\tau(t))+d e_{\xi}^{T}(t-\tau(t))\left[\left(H_{s}^{T} H_{s}\right) \otimes I_{2}\right] \\
& \cdot e_{\xi}(t-\tau(t)) .
\end{aligned}
$$

By Newton-Leibniz formula,

$$
e_{\xi}(t-\tau(t))=e_{\xi}(t)-\int_{t-\tau(t)}^{t} \dot{e}_{\xi}(r) \mathrm{d} r
$$


and noting that $2 x^{T} y \leq x^{T} A^{-1} x+y^{T} A y$ hold for any appropriate positive definite matrix $A$, we have

$$
\begin{aligned}
& -2 e_{\xi}^{T}(t)\left[\left(\Xi H_{s}\right) \otimes I_{2}\right] e_{\xi}(t-\tau(t)) \\
& \leq-2 e_{\xi}^{T}(t)\left[\left(\Xi H_{s}\right) \otimes I_{2}\right] e_{\xi}(t) \\
& \quad+\int_{t-\tau(t)}^{t} 2 e_{\xi}^{T}(t)\left[\left(\Xi H_{s}\right) \otimes I_{2}\right] \dot{e}_{\xi}(r) \mathrm{d} r \\
& \leq-e_{\xi}^{T}(t)\left[\left(\Xi H_{s}+H_{s}^{T} \Xi\right) \otimes I_{2}\right] e_{\xi}(t) \\
& +d e_{\xi}^{T}(t)\left[\left(\Xi H_{s} H_{s}^{T} \Xi\right) \otimes I_{2}\right] e_{\xi}(t) \\
& +\int_{t-\tau(t)}^{t} \dot{e}_{\xi}^{T}(r) \dot{e}_{\xi}(r) \mathrm{d} r .
\end{aligned}
$$

Consequently,

$$
\begin{gathered}
V(t) \leq-\left[\lambda_{\min }\left(\Xi H_{s}+H_{s}^{T} \Xi\right)-d \lambda_{\max }\left(\Xi H_{s} H_{s}^{T} \Xi\right)\right. \\
\left.-\gamma \lambda_{\max }\left(H_{s}^{T} H_{s}\right)\right] e_{\xi}^{T}(t) e_{\xi}(t) \\
-\left[\gamma\left(1-d_{1}\right) \lambda_{\min }\left(H_{s}^{T} H_{s}\right)\right. \\
\left.-d \lambda_{\max }\left(H_{s}^{T} H_{s}\right)\right] e_{\xi}^{T}(t-\tau(t)) e_{\xi}(t-\tau(t)) .
\end{gathered}
$$

Then, a sufficient condition for $\dot{V}(t)<0$ is

$$
\begin{aligned}
& \lambda_{\min }\left(\Xi H_{s}+H_{s}^{T} \Xi\right)-d \lambda_{\max }\left(\Xi H_{s} H_{s}^{T} \Xi\right) \\
& \quad-\gamma \lambda_{\max }\left(H_{s}^{T} H_{s}\right)>0, \\
& \gamma\left(1-d_{1}\right) \lambda_{\min }\left(H_{s}^{T} H_{s}\right)-d \lambda_{\max }\left(H_{s}^{T} H_{s}\right)>0,
\end{aligned}
$$

which leads to

$$
\begin{aligned}
& \frac{d \lambda_{\max }\left(H_{s}^{T} H_{s}\right)}{\left(1-d_{1}\right) \lambda_{\min }\left(H_{s}^{T} H_{s}\right)} \\
& <\gamma<\frac{\lambda_{\min }\left(\Xi H_{s}+H_{s}^{T} \Xi\right)-d \lambda_{\max }\left(\Xi H_{s} H_{s}^{T} \Xi\right)}{\lambda_{\max }\left(H_{s}^{T} H_{s}\right)} .
\end{aligned}
$$

Then, one can deduce that

$$
d<\frac{\left(1-d_{1}\right) \lambda_{\min }\left(\Xi H_{s}+H_{s}^{T} \Xi\right)}{\lambda_{\max }^{2}\left(H_{s}^{T} H_{s}\right) / \lambda_{\min }\left(H_{s}^{T} H_{s}\right)+\left(1-d_{1}\right) \lambda_{\max }\left(\Xi H_{s} H_{s}^{T} \Xi\right)}
$$

for given $d_{1}<1$. This completes the proof.

Remark 3. As we all know, the communication latency is a key factor that cannot be ignored in the smart microgrid control problem since many cases have proven that neglect of the communication latency will lead to serious and unpredictable consequences. When analyzing the nonlinear systems containing time-varying communication latency, most existing literatures presented some delay independent stability conditions or just require that the delay is sufficiently small. However, in order to investigate the upper bound of the amount of time-varying delay, this paper constructs a Lyapunov-Krasovskii functional (i.e., $V(x)$ ) with delayrelated parameters. It is worthy pointing out that, for the presented nonlinear system with directed and switching topology, the asymmetry of the adjacency matrix and the nonlinearity of the controlled systems will increase the difficulty to construct $V(x)$ and analyze its stability. By using the special matrix theory, inequality theory, and theory of delay differential equations, this paper solves the above problems.

3.2. Active Power Sharing. It is [26] that has proposed a method on how to choose the frequency droop coefficients and nominal setpoints to keep DGs/SGs realizing the active power sharing according to their power ratings. This control goal is analyzed in many literatures and spread to actual applications [5]. The proposed proportional power sharing method also can guarantee that storage devices in charging mode, that is, $P_{i}^{\text {nom,stor }}$ for some $i \in N$, are charged proportionally.

In the following, the DGs/SGs proportionally share their power in terms of their power ratings $P_{i}^{\max }$ if the gains $K_{i}^{P}$ and setpoints $P_{i}^{\text {nom }}$ are chosen such that, for all $i=1, \ldots, N$, the condition $K_{i}^{P} P_{i}=K_{j}^{P} P_{j}$ is standard in the microgrid research:

$$
\frac{P_{i}}{P_{i}^{\max }}=\frac{P_{j}}{P_{j}^{\max }}
$$

where $P_{i}^{\max }$ and $P_{j}^{\max }$ are the instantaneous maximum capacity of $P_{i}$ and $P_{j}$ and all the $P_{i}^{\max }$ and $P_{j}^{\max }$ are assumed to have the same sign. The active powers of DGs/SGs are able to be controlled according to condition (19) and droop control (2) the active power sharing proportionally. Condition (19) that also has a guiding significance achieves the reactive power sharing.

\section{Simulation Results and Discussion}

In this section, the effectiveness of the proposed distributed control algorithms will be verified by simulating an islanded lossy microgrid in MATLAB. Based on the above analysis for the identity property on dynamics of DGs and SGs, without loss of generality, five DGs have been chosen to the test system. Figure 4 shows the basic diagram of the test system which consists of five DGs. The specifications of the DGs, lines, and loads are summarized in Table 1 . Note that its communication topology can be abstracted as digraph $\mathscr{G}_{1}$ shown in Figure 5. The associated Laplacian matrix of $\mathscr{G}_{1}$ is

$$
\begin{aligned}
& L_{1} \\
& =\left(\begin{array}{ccccc}
0.005 & 0 & 0 & 0 & -0.005 \\
-0.005 & 0.005 & 0 & 0 & 0 \\
0 & -0.005 & 0.005 & 0 & 0 \\
0 & 0 & -0.005 & 0.005 & 0 \\
0 & 0 & 0 & -0.005 & 0.005
\end{array}\right),
\end{aligned}
$$


TABLE 1: Parameter values for test system.

\begin{tabular}{lccccccccc}
\hline & $\mathrm{DG}_{1}$ & \multicolumn{2}{c}{$\mathrm{DG}_{2}$} & \multicolumn{2}{c}{$\mathrm{DG}_{3}$} & \multicolumn{2}{c}{$\mathrm{DG}_{4}$} & $\mathrm{DG}_{5}$ \\
\hline$V_{\mathrm{DC}}$ & $680(\mathrm{~V})$ & $V_{\mathrm{DC}}$ & $1000(\mathrm{~V})$ & $V_{\mathrm{DC}}$ & $800(\mathrm{~V})$ & $V_{\mathrm{DC}}$ & $750(\mathrm{~V})$ & $V_{\mathrm{DC}}$ & $700(\mathrm{~V})$ \\
\hline$k^{P}$ & $0.8 \times 10^{-4}$ & $k^{P}$ & $1.6 \times 10^{-4}$ & $k^{P}$ & $1.4 \times 10^{-4}$ & $k^{P}$ & $1.2 \times 10^{-4}$ & $k^{P}$ & $1.0 \times 10^{-4}$ \\
\hline$k^{\mathrm{Q}}$ & $1.6 \times 10^{-3}$ & $k^{\mathrm{Q}}$ & $3.7 \times 10^{-3}$ & $k^{\mathrm{Q}}$ & $3.2 \times 10^{-3}$ & $k^{\mathrm{P}}$ & $2.8 \times 10^{-3}$ & $k^{\mathrm{Q}}$ & $2.1 \times 10^{-3}$ \\
\hline$R_{c}$ & $0.001(\Omega)$ & $R_{c}$ & $0.001(\Omega)$ & $R_{c}$ & $0.001(\Omega)$ & $R_{c}$ & $0.001(\Omega)$ & $R_{c}$ & $0.001(\Omega)$ \\
\hline$L_{c}$ & $0.2(\mathrm{mH})$ & $L_{c}$ & $0.2(\mathrm{mH})$ & $L_{c}$ & $0.2(\mathrm{mH})$ & $L_{c}$ & $0.2(\mathrm{mH})$ & $L_{c}$ & $0.2(\mathrm{mH})$ \\
\hline Load $_{1}$ & Load $_{2}$ & $\mathrm{Load}_{3}$ & $\mathrm{Load}_{4}$ & $R_{1}+L_{1}$ & $R_{2}+L_{2}$ & $R_{3}+L_{3}$ & $R_{4}+L_{4}$ & $V_{\mathrm{MG}}$ & $f_{\mathrm{MG}}$ \\
\hline $13.5(\mathrm{Kw})$ & $18.5(\mathrm{Kw})$ & $13.5(\mathrm{Kw})$ & $14(\mathrm{Kw})$ & $4+j 1.95(\Omega)$ & $2.2+j 1.4(\Omega)$ & $1.5+j 0.7(\Omega)$ & $1.2+j 0.3(\Omega)$ & $380(\mathrm{~V})$ & $50(\mathrm{~Hz})$ \\
$12(\mathrm{KvAr})$ & $14(\mathrm{KvAr})$ & $12(\mathrm{KvAr})$ & $13(\mathrm{KvAr})$ & & & & & & \\
\hline
\end{tabular}

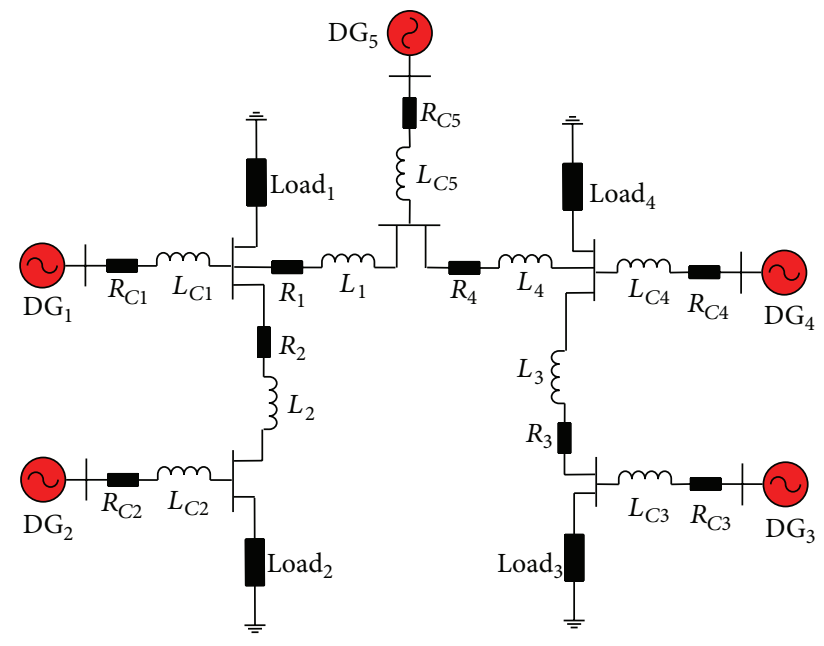

FIgURE 4: The fixed communication network of DGs.

and the corresponding leader adjacency matrix can be chosen as $B_{1}=\operatorname{diag}\{0.005,0,0.005,0,0\}$.

In the following simulation, the time-varying delay $\tau(t)$ is always taken as $\tau(t)=(1+0.1 \sin (t)) / 32$; then $\tau(t)<$ $d=0.0344$ and $\dot{\tau}(t)<d_{1}=0.0031<1$. For the fixed digraph $\mathscr{G}_{1}$, by simple calculation, one can choose $\Xi=\operatorname{diag}\{0.2,0.2,0.2,0.2,0.2\}$ and then yield $\lambda_{\min }\left(\Xi H_{1}+\right.$ $\left.H_{1}^{T} \Xi\right)=5.2717 \times 10^{-4}, \lambda_{\max }\left(\Xi H_{1} H_{1}^{T} \Xi\right)=6.4856 \times 10^{-6}$, $\lambda_{\text {max }}\left(H_{1}^{T} H_{1}\right)=1.6214 \times 10^{-4}$, and $\lambda_{\min }\left(H_{1}^{T} H_{1}\right)=2.1074 \times$ $10^{-6}$ with $H_{1}=L_{1}+B_{1}$. Therefore, the delay constraint condition in Theorem 2 can be verified as

$d=0.0031$

$$
\begin{aligned}
& <\frac{\left(1-d_{1}\right) \lambda_{\min }\left(\Xi H_{1}+H_{1}^{T} \Xi\right)}{\lambda_{\max }^{2}\left(H_{1}^{T} H_{1}\right) / \lambda_{\min }\left(H_{1}^{T} H_{1}\right)+\left(1-d_{1}\right) \lambda_{\max }\left(\Xi H_{1} H_{1}^{T} \Xi\right)} \\
& =0.0421 .
\end{aligned}
$$

For the switching digraph $\Gamma$ shown in Figure 5, one can verify the above conditions similarly.

(1) Case 1 (fixed topology). Consider a directed communication network with five DGs as shown in $\mathscr{G}_{1}$. Figures 6 and 7 show the state evolution processes of frequency,

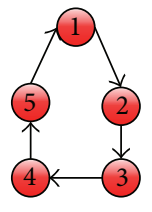

$\mathscr{G}_{1}$

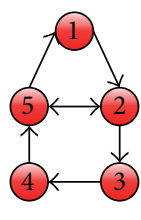

$\mathscr{G}_{2}$

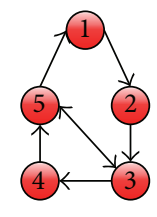

$\mathscr{G}_{3}$

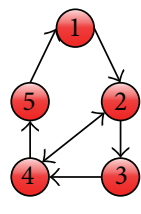

$\mathscr{G}_{4}$

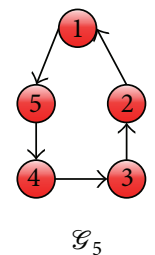

FIgURE 5: The switching communication digraph topology $\Gamma$. corresponding to active power with and without delay, respectively. It is assumed that the demands of loads increase suddenly the islanded operation microgrid at $t=0.4 \mathrm{~s}$. As seen in Figure 6(a), after islanding, each DG's frequency $\omega$ goes to different values less than reference value $\omega^{\text {ref }}=2 \pi f=$ $314 \mathrm{rad} / \mathrm{s}$. Then the primary control and secondary control are applied at $t=0.5 \mathrm{~s}$ and $t=1 \mathrm{~s}$, respectively. Due to the droop control technique, each DG's frequency $\omega$ converges to stability value still less than reference value. However, the secondary control restores the operating frequency to synchronize their reference value after $1.6 \mathrm{~s}$.

Comparing Figures 6 and 7, respectively, one finds that the existence of communication delay postpones the convergence speed of frequency.

(2) Case 2 (switching topology). Consider a directed network with switching topology $\Gamma=\left\{\mathscr{G}_{1}, \ldots, \mathscr{G}_{5}\right\}$ as shown in Figure 5. The controlled system begins at the state $\mathscr{G}_{1}$ and switches at $\Delta t=0.001$ to the next stage with the following switching order: $\left\{\mathscr{G}_{1}, \ldots, \mathscr{G}_{5}, \mathscr{G}_{1}, \ldots\right\}$, where the switching signal $1 \leq i \leq 5$. All the weights are set to be 0.005 , and the corresponding leader adjacency matrix for $\mathscr{G}_{2}$ to $\mathscr{G}_{5}$ is chosen as $B_{2}=\operatorname{diag}\{0,0.005,0,0.005,0\}, B_{3}=$ $\operatorname{diag}\{0,0,0.005,0,0.005\}, B_{4}=\operatorname{diag}\{0.005,0,0,0.005,0\}$, and $B_{5}=\operatorname{diag}\{0,0,0,0.005,0.005\}$. The associated system parameters are similar to the fixed topology case.

Figures 8 and 9 show the state evolution processes of frequency, corresponding to active power with and without delay, respectively. Comparing Figures 6-7 and Figures 8-9, it is not difficult to find that the evolution curves in Figures 8-9 contain some slight oscillation phases. It is because that the controlled system in this case contains frequent switching signals. Similarly, the communication delay also postpones the convergence speed of the whole switching system. 


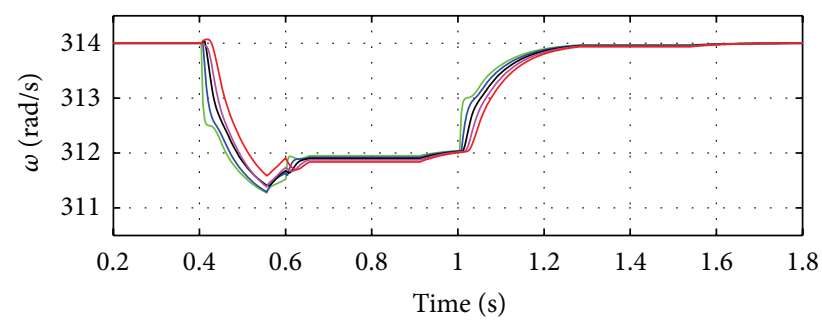

- DG 1

- DG 2

- DG 3

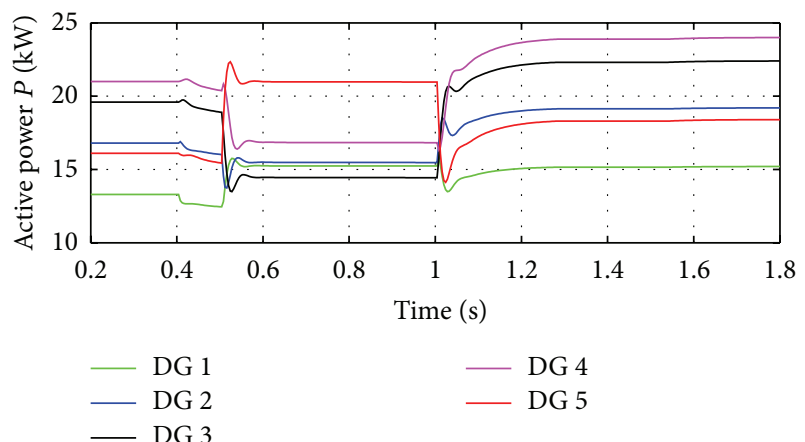

(b)

FIGURE 6: The state evolution processes of frequency, active power with delay under $\mathscr{G}_{1}$.
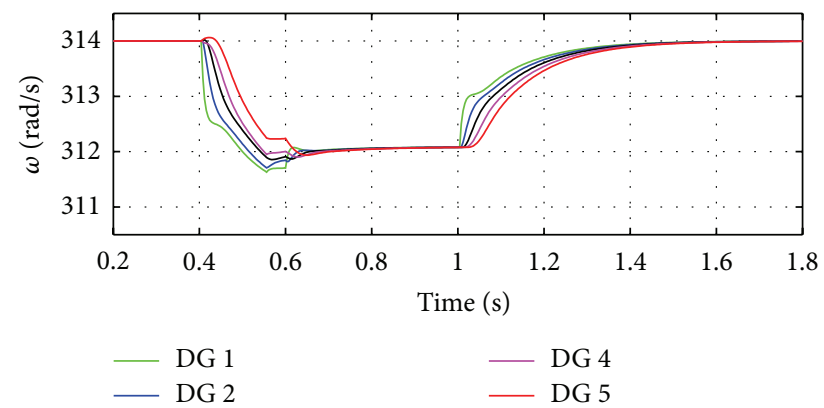

- DG 3

(a)
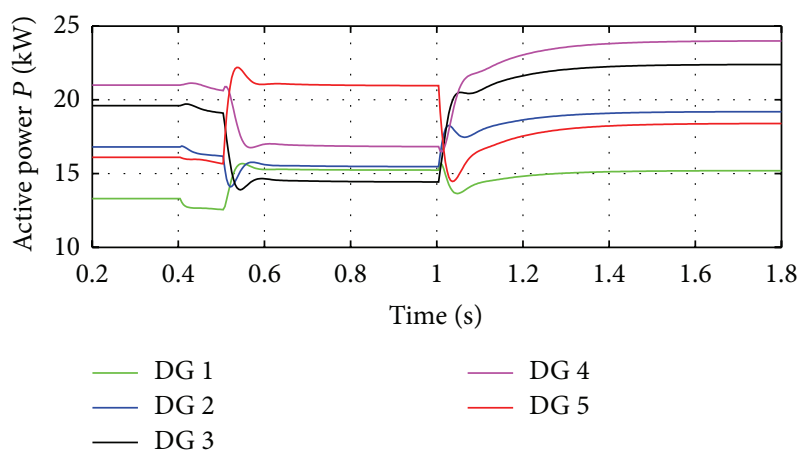

(b)

FIGURE 7: The state evolution processes of frequency, active power without delay under $\mathscr{G}_{1}$.

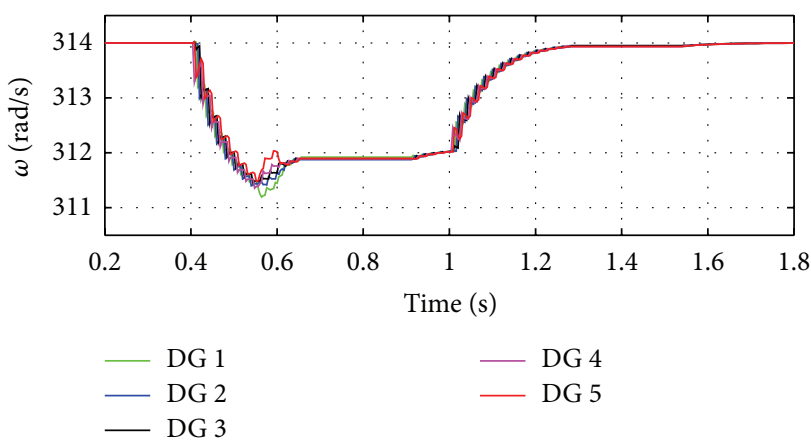

(a)

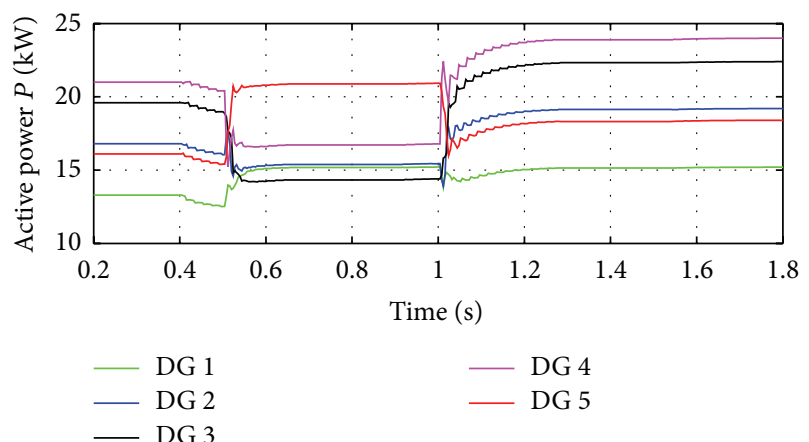

(b)

FIGURE 8: The state evolution processes of frequency, active power with delay under $\Gamma$.

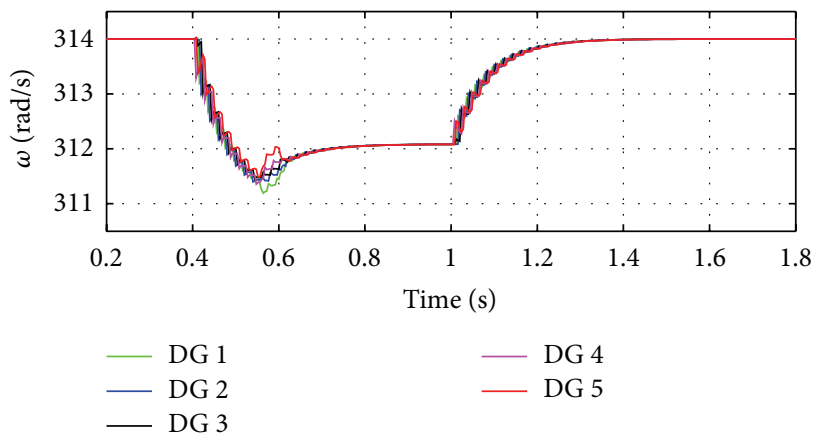

(a)

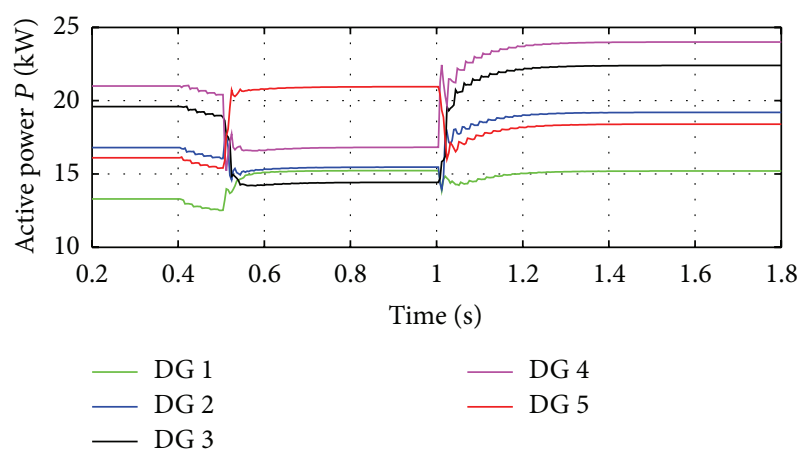

(b)

FIGURE 9: The state evolution processes of frequency, active power without delay under $\Gamma$. 


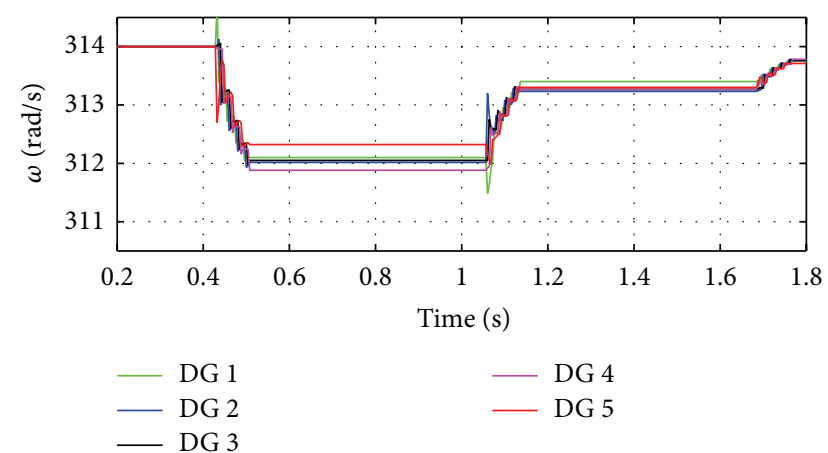

(a)

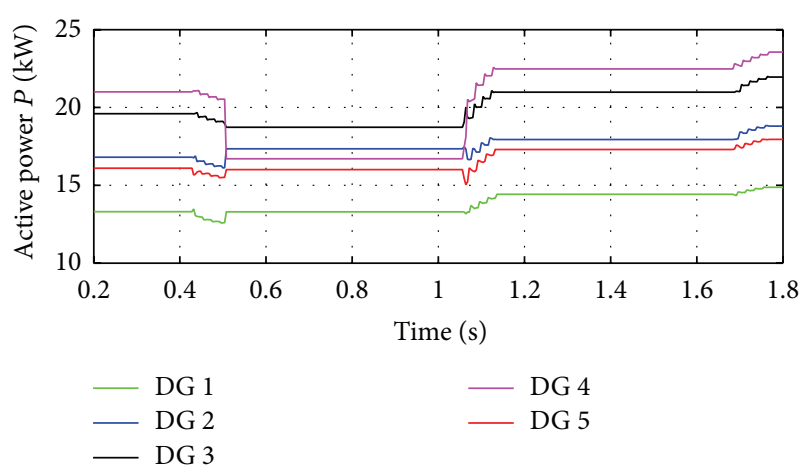

(b)

FIGURE 10: The state evolution processes of frequency, active power with large delay under $\Gamma$.

However, if we take the large delay $\tau(t)=(2+$ $\sin (t)) / 32$ which does not satisfy the constraint conditions in Theorem 2, the proposed distributed control algorithms may be ineffective. As shown in Figure 10, the state evolution curves do not converge to the desired values in switching case.

\section{Conclusion}

In this paper, a distributed cooperative control scheme for hybrid lossy microgrids has been proposed. In this method, a distributed secondary control encompasses each DG/SG local controller and the communication system; that is, each DG/SG only needs to communicate with its neighboring DGs/SGs intermittently even though the communication delays may be time-varying. The designed distributed control is able to synchronize the frequency of hybrid lossy microgrids to the desired state $(50 \mathrm{~Hz})$ in a distribution switching network under the existence of time-varying communication delays. All the frequency of DGs/SGs can be synchronized to the desired value in both fixed and switching distributed networks. Meanwhile, numerical simulations show higher robustness in front large communication latency.

\section{Conflict of Interests}

The authors declare that there is no conflict of interests regarding the publication of this paper.

\section{Acknowledgments}

This work was supported by National Key Technology Support Program of China under Grant no. 2013BAA01B01; by the National Natural Science Foundation of China under Grant nos. 61403133, 61374064, and 61304152; by the Young Teachers Growth Plan of Hunan University under Grant no. 531107040651; by the Postdoctoral Foundation of China under Grant no. 2013M540627; by the Postdoctoral Special Foundation of Hunan Province under Grant no. 2013RS4042; by the Natural Science Foundation of Hunan Province under Grant no. 14JJ3051; by the Doctoral Fund of Ministry of Education of China under Grant no. 20130161120016; by the Fundamental Research Funds for the Central Universities no. 2014208020201.

\section{References}

[1] N. Pogaku, M. Prodanović, and T. C. Green, "Modeling, analysis and testing of autonomous operation of an inverter-based microgrid," IEEE Transactions on Power Electronics, vol. 22, no. 2, pp. 613-625, 2007.

[2] J. G. Lai, H. Zhou, W. S. Hu, D. G. Zhou, and L. Zhong, "Smart demand response based on smart homes," Mathematical Problems in Engineering. In press.

[3] H. Farhangi, "The path of the smart grid," IEEE Power and Energy Magazine, vol. 8, no. 1, pp. 18-28, 2010.

[4] J. M. Guerrero, J. C. Vasquez, J. Matas, L. G. de Vicuña, and M. Castilla, "Hierarchical control of droop-controlled AC and DC microgrids-a general approach toward standardization," IEEE Transactions on Industrial Electronics, vol. 58, no. 1, pp. 158-172, 2011.

[5] Q.-C. Zhong, "Robust droop controller for accurate proportional load sharing among inverters operated in parallel," IEEE Transactions on Industrial Electronics, vol. 60, no. 4, pp. 12811290, 2013.

[6] Y. A.-R. I. Mohamed and E. F. El-Saadany, "Adaptive decentralized droop controller to preserve power sharing stability of paralleled inverters in distributed generation microgrids," IEEE Transactions on Power Electronics, vol. 23, no. 6, pp. 2806-2816, 2008.

[7] M. Savaghebi, A. Jalilian, J. C. Vasquez, and J. M. Guerrero, "Secondary control for voltage quality enhancement in microgrids," IEEE Transactions on Smart Grid, vol. 3, no. 4, pp. 1893-1902, 2012.

[8] M. Savaghebi, A. Jalilian, J. C. Vasquez, and J. M. Guerrero, "Secondary control scheme for voltage unbalance compensation in an Islanded droop-controlled microgrid," IEEE Transactions on Smart Grid, vol. 3, no. 2, pp. 797-807, 2012.

[9] J. Schiffer, R. Ortegab, A. Astolfic et al., "Conditions for stability of droop-controlled inverter-based microgrids," Automatica, vol. 50, no. 10, pp. 2457-2469, 2014.

[10] Q. Shafiee, C. Stefanovic, T. Dragicevic, P. Popovski, J. C. Vasquez, and J. M. Guerrero, "Robust networked control scheme for distributed secondary control of islanded microgrids," IEEE Transactions on Industrial Electronics, vol. 61, no. 10, pp. 5363-5374, 2014.

[11] H. Bouattour, J. W. Simpson-Porco, F. Dörfler, and F. Bullo, "Further results on distributed secondary control in microgrids," in Proceedings of the 52nd IEEE Conference on Decision and Control (CDC '13), pp. 1514-1519, December 2013. 
[12] P. N. Vovos, A. E. Kiprakis, A. R. Wallace, and G. P. Harrison, "Centralized and distributed voltage control: impact on distributed generation penetration," IEEE Transactions on Power Systems, vol. 22, no. 1, pp. 476-483, 2007.

[13] A. G. Tsikalakis and N. D. Hatziargyriou, "Centralized control for optimizing microgrids operation," in Proceedings of the IEEE PES General Meeting: The Electrification of Transportation and the Grid of the Future, pp. 1-8, July 2011.

[14] A. Vaccaro, G. Velotto, and A. F. Zobaa, "A decentralized and cooperative architecture for optimal voltage regulation in smart grids," IEEE Transactions on Industrial Electronics, vol. 58, no. 10, pp. 4593-4602, 2011.

[15] G. A. Pagani and M. Aiello, "Towards decentralization: a topological investigation of the medium and low voltage grids," IEEE Transactions on Smart Grid, vol. 2, no. 3, pp. 538-547, 2011.

[16] J. G. Lai, S. H. Chen, X. Q. Lu, and H. Zhou, "Formation tracking for nonlinear mulit-agent systems with delays and noises disturbance," Asian Journal of Control, vol. 17, no. 3, pp. 1-13, 2015.

[17] J. Lai, S. Chen, and X. Lu, "Tracking consensus of nonlinear MASs with asymmetric communication delays in noisy environments," Communications in Nonlinear Science and Numerical Simulation, vol. 19, no. 7, pp. 2334-2344, 2014.

[18] Z. H. Guan, Z. W. Liu, G. Feng, and Y. W. Wang, "Synchronization of complex dynamical networks with time-varying delays via impulsive distributed control," IEEE Transactions on Circuits and Systems. I. Regular Papers, vol. 57, no. 8, pp. 2182-2195, 2010.

[19] Z.-W. Liu, Z.-H. Guan, X. M. Shen, and G. Feng, "Consensus of multi-agent networks with aperiodic sampled communication via impulsive algorithms using position-only measurements," IEEE Transactions on Automatic Control, vol. 57, no. 10, pp. 2639-2643, 2012.

[20] X. Lu, R. Lu, S. Chen, and J. Lu, "Finite-time distributed tracking control for multi-agent systems with a virtual leader," IEEE Transactions on Circuits and Systems. I. Regular Papers, vol. 60, no. 2, pp. 352-362, 2013.

[21] X. Q. Lu, S. H. Chen, and J. H. Lü, "Finite-time tracking for double-integrator multi-agent systems with bounded control input," IET Control Theory \& Applications, vol. 7, no. 11, pp. 15621573, 2013.

[22] K. Tanaka, M. Oshiro, S. Toma et al., "Decentralised control of voltage in distribution systems by distributed generators," IET Generation, Transmission and Distribution, vol. 4, no. 11, pp. 1251-1260, 2010.

[23] H. Zhou, Z.-H. Wang, Z.-W. Liu, W. S. Hu, and G. P. Liu, "Containment control for multi-agent systems via impulsive algorithms without velocity measurements," IET Control Theory \& Applications, vol. 8, no. 17, pp. 2033-2044, 2014.

[24] C. H. Zhao, U. Topcu, N. Li, and S. Low, "Design and stability of load-side primary frequency control in power systems," IEEE Transactions on Automatic Control, vol. 59, no. 5, pp. 1177-1189, 2014.

[25] H. Bouattour, J. W. Simpson-Porco, F. Dorfler, and F. Bullo, "Further results on distributed secondary control in microgrids," in Proceedings of the IEEE 52nd Annual Conference on Decision and Control (CDC '13), pp. 1514-1519, Firenze, Italy, December 2013.

[26] J. W. Simpson-Porco, F. Dörfler, and F. Bullo, "Synchronization and power sharing for droop-controlled inverters in islanded microgrids," Automatica, vol. 49, no. 9, pp. 2603-2611, 2013.

[27] H. Zhang, F. L. Lewis, and A. Das, "Optimal design for synchronization of cooperative systems: state feedback, observer and output feedback," IEEE Transactions on Automatic Control, vol. 56, no. 8, pp. 1948-1952, 2011.

[28] H. H. Xin, Z. H. Qu, J. Seuss, and A. Maknouninejad, "A self-organizing strategy for power flow control of photovoltaic generators in a distribution network," IEEE Transactions on Power Systems, vol. 26, no. 3, pp. 1462-1473, 2011.

[29] H. Liang, B. J. Choi, W. Zhuang, and X. Shen, "Stability enhancement of decentralized inverter control through wireless communications in microgrids," IEEE Transactions on Smart Grid, vol. 4, no. 1, pp. 321-331, 2013.

[30] Q. Shafiee, J. M. Guerrero, and J. C. Vasquez, "Distributed secondary control for islanded microgrids-a novel approach," IEEE Transactions on Power Electronics, vol. 29, no. 2, pp. 10181031, 2014.

[31] J. Schiffera, D. Goldin, J. Raisch et al., "Synchronization of droop-controlled microgrids with distributed rotational and electronic generation," in Proceedings of the IEEE 52nd Annual Conference on Decision and Control (CDC '13), pp. 2334-2339, 2013.

[32] X. Lu, F. Austin, and S. Chen, "Flocking in multi-agent systems with active virtual leader and time-varying delays coupling," Communications in Nonlinear Science and Numerical Simulation, vol. 16, no. 2, pp. 1014-1026, 2011. 


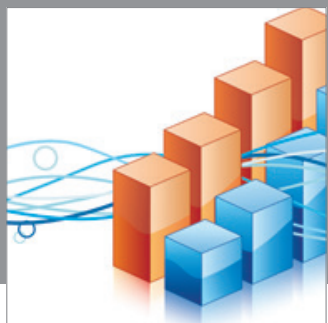

Advances in

Operations Research

mansans

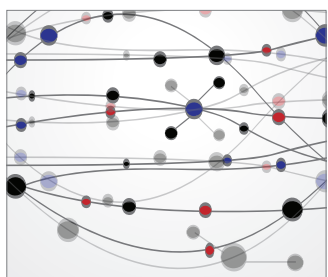

The Scientific World Journal
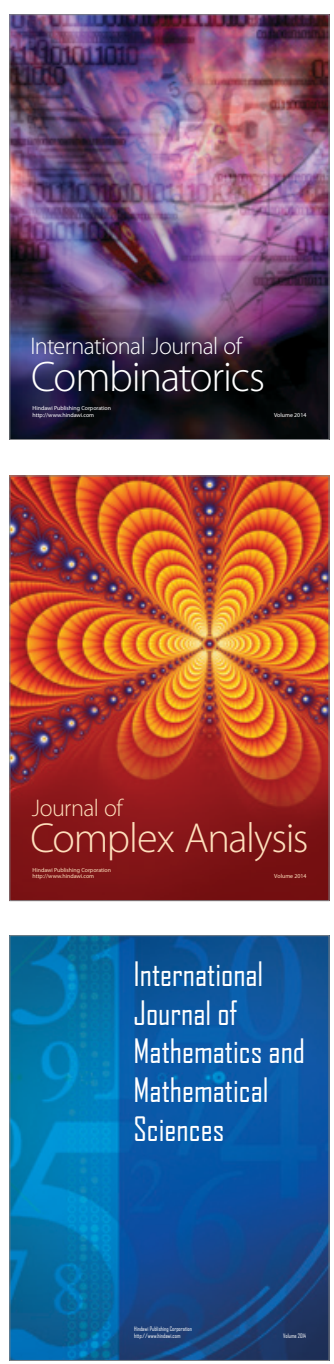
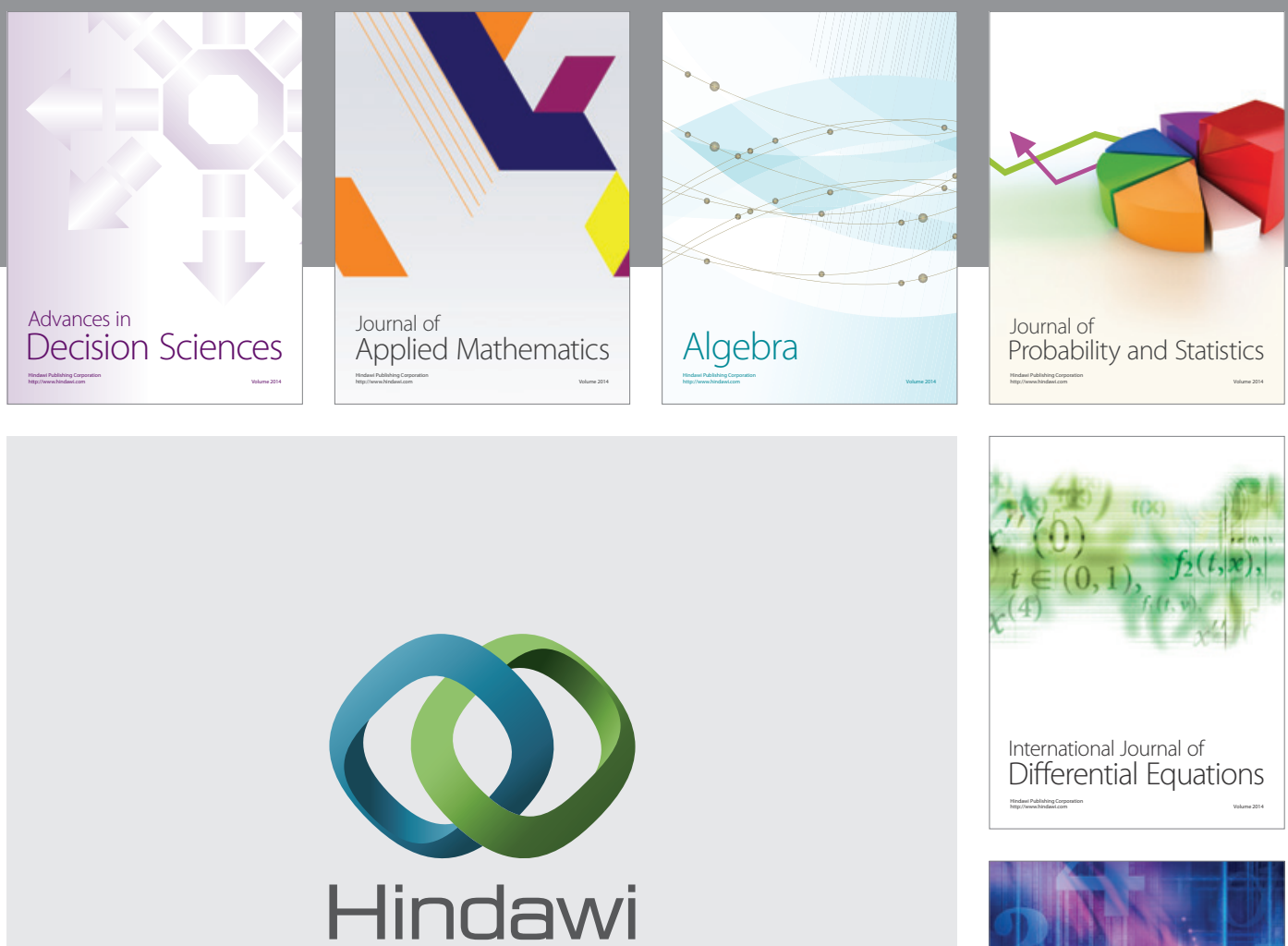

Submit your manuscripts at http://www.hindawi.com
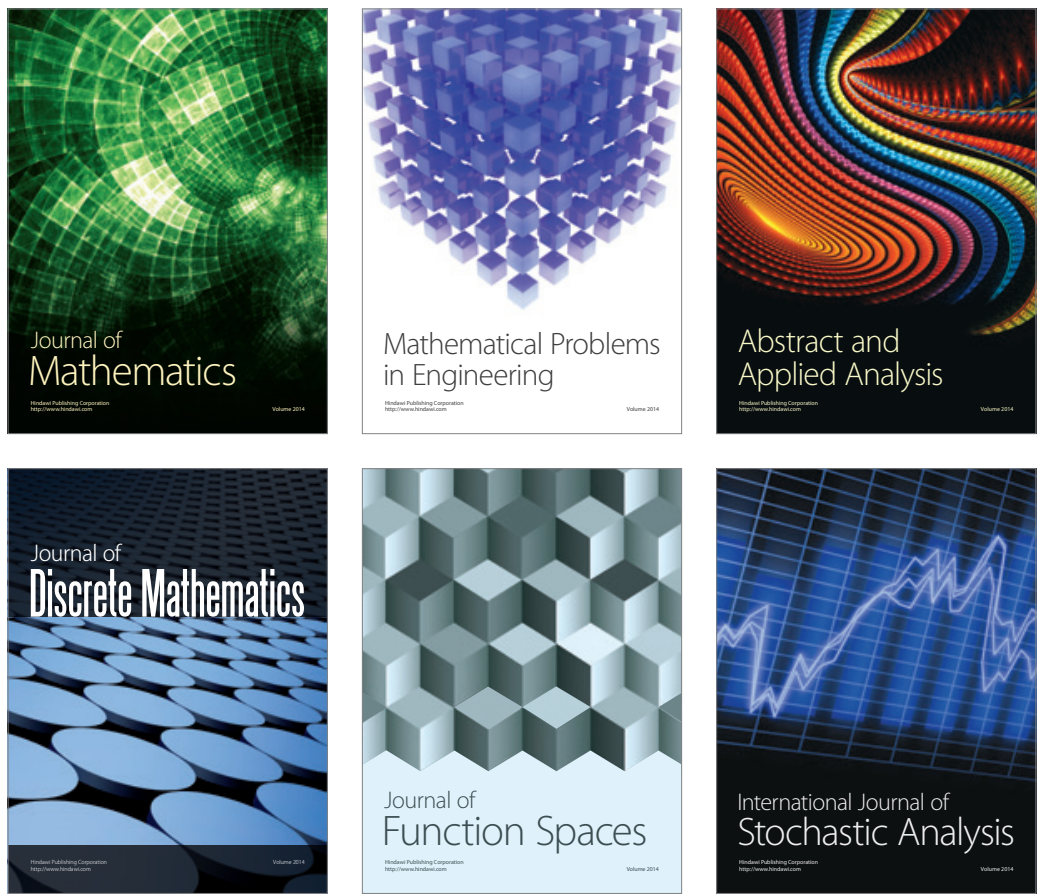

Journal of

Function Spaces

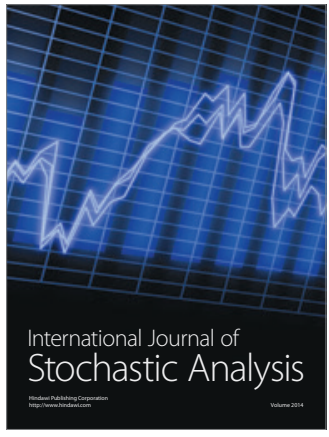

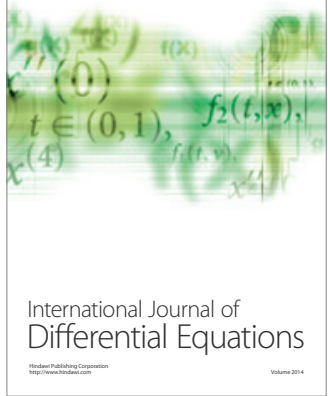
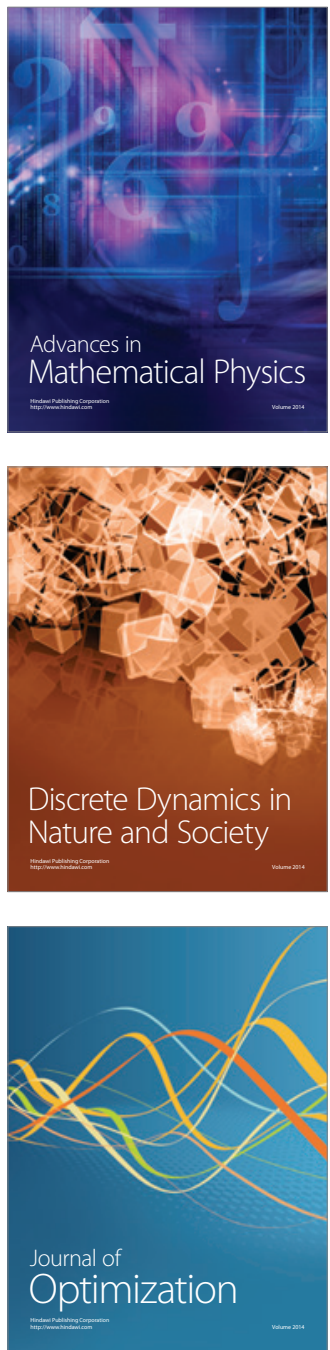